\title{
Omni-directional catadioptric vision for soccer robots
}

\author{
Pedro Lima ${ }^{\mathrm{a}, *}$, Andrea Bonarini ${ }^{\mathrm{b}, 1,2}$, Carlos Machado $^{\mathrm{c}}$, Fabio Marchese ${ }^{\mathrm{d}, 1,3}$, \\ Carlos Marques ${ }^{\mathrm{a}, 4}$, Fernando Ribeiro $^{\mathrm{c}}$, Domenico Sorrenti ${ }^{\mathrm{d}, 1,3}$ \\ ${ }^{a}$ Instituto de Sistemas e Robótica, Instituto Superior Técnico, Av. Rovisco Pais, 1, 1049-001 Lisboa, Portugal \\ b Politecnico di Milano AI and Robotics Project, Dipartimento di Elettronica e Informazione, \\ Politecnico di Milano, Piazza Leonardo da Vinci 32, I-20133 Milano, Italy \\ c Grupo de Automação e Robótica, Departamento de Electrónica Industrial, \\ Universidade do Minho, Campos de Azurém, 4800 Guimarães, Portugal \\ d Dipartimento di Informatica, Sistemistica e Comunicazione, Università degli Studi di Milano - Bicocca, \\ via Bicocca degli Arcimboldi 8, I-20126 Milano, Italy
}

\begin{abstract}
\end{abstract}
This paper describes the design of a multi-part mirror catadioptric vision system and its use for self-localization and detection of relevant objects in soccer robots. The mirror and associated algorithms have been used in robots participating in the middle-size league of RoboCup — The World Cup of Soccer Robots. (C) 2001 Published by Elsevier Science B.V.

Keywords: Omni-directional vision; Catadioptric vision; Soccer robots; Self-localization; Virtual sensors; Hough transform
There are many different ways of assembling a camera on a robot:

- Fixed camera pointing to the front of the robot: In this case, a typical image that can be seen from the robot is depicted in Fig. 1. The main disadvantage of this solution results from the limited amount of available information, and from the increasing occurrence of occlusions of the scene background due to nearby objects.

- Motorized camera: This results from assembling the camera on a structure linked to a motor. The field of view is increased by moving the camera up and down (tilt) and/or left and right (pan). A major problem is also finding an effective way of coordinating robot and camera motion.

- More than one camera: More than one camera can be used, on one hand, to achieve stereo vision and determine the distance to relevant objects, on the other hand, to watch different spots around the robot. This is, however, a costly solution. Further- 


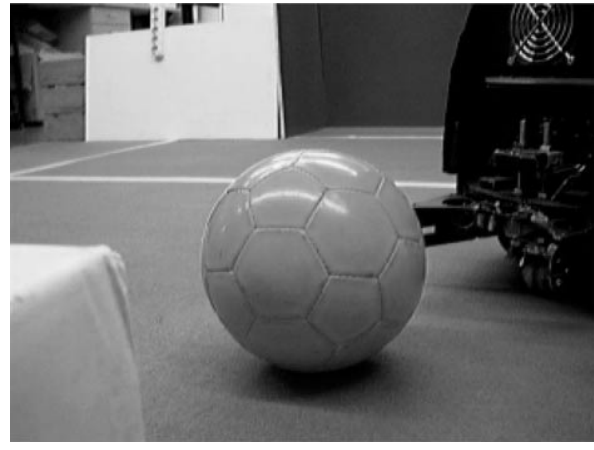

Fig. 1. Image seen by a robot with a camera pointing to its front.

more, reliability is decreased due to the increasing number of devices and the power consumption can be considerable.

- Fixed camera pointed to one or more mirrors: This belongs to the class of solutions known as catadioptric vision systems. One important example are the omni-directional vision systems, based on a camera pointing upwards to a convex mirror (see Fig. 2(a)). The main disadvantage of omni-directional catadioptric vision systems is the distortion, on the image, of the shape of relevant objects in the observed scene. Nevertheless, if the information to be

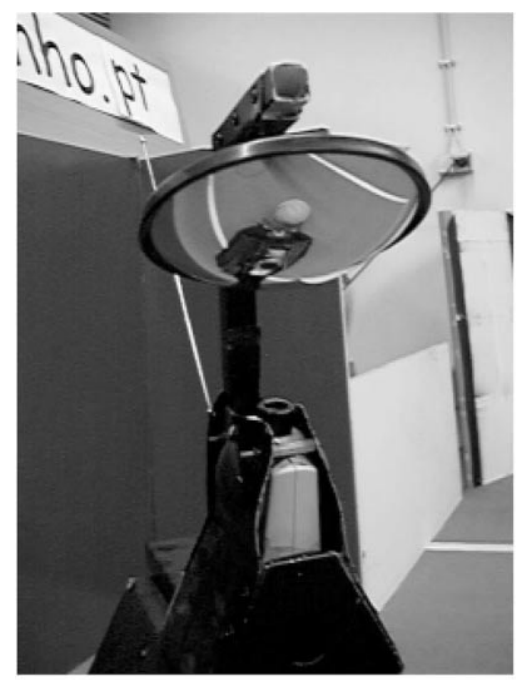

(a) extracted from the image is only the relative orientation, distortion is irrelevant, since the angles between radial lines are preserved [2]. Different mirror profiles can be used, such as conic mirrors, parabolic mirrors or spherical mirrors, to name a few, each one with a different type of distortion. When the mirror profile is precisely known, the image can be unwarped with a suitable transformation, i.e., the inverse of the transformation performed by the mirror. Another solution is to design mirrors which unwarp the image directly, saving CPU time $[3,4]$. Another potential problem is the support type used for the mirror. The support must be carefully chosen, since it may introduce further distortion and/or occlusion (e.g., in Fig. 2a, the image is partially occluded by the supporting structure of the mirror and by the camera itself). An example of image captured by an omni-directional mirror can be seen in Fig. 2(b).

This paper focusses on the design and use of omni-directional catadioptric vision systems for soccer robots. In the RoboCup-Soccer competitions, the field features are mainly distinguishable by their color (e.g., the field is green with white lines, the goals are blue and yellow, the ball is orange), hence vision is a sensor naturally shared by all participant teams.

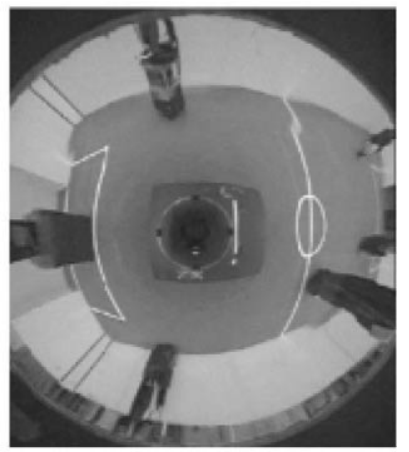

(b)

Fig. 2. (a) Omni-directional catadioptric vision system consisting of a camera and a parabolic mirror; (b) an image taken by a conical-spherical sensor. 
In the middle-size league of RoboCup-Soccer, the teams are composed of fully autonomous robots, with no global view of the field and most, if not all, processing done on board. Among those, an increasing number of teams is using omni-directional catadioptric vision, so that many different important environment features can be seen simultaneously whenever an image frame is acquired. In this paper, we describe the approach to omni-directional vision in the middle-size league of RoboCup-Soccer by three such teams:

- ART team, partially represented here by the Politecnico di Milano and the Università degli Studi di Milano - Bicocca, Italy;

- Minho, from the University of Minho, Portugal;

- ISocRob, from the Instituto Superior Técnico, Portugal.

Three main topics covered by the paper are the following:

- The design of a multi-part omni-directional mirror.

- Virtual sensors to extract important environment features from the image.

- Omni-directional vision-based self-localization.

Each of the above groups concentrated on one of the topics (listed in the same order). This paper aims at demonstrating that an integration of the work done, based on the described catadioptric vision system with a multi-part mirror, is possible. Nevertheless, the results presented were obtained with mirrors separately designed by the different groups, each corresponding to particular parts of the multi-part mirror.

In the literature, different mirror geometries have been proposed [5,6] and even in RoboCup-Soccer middle-size league, some teams already used mirrors [7-9] with profiles other than the original conical one [5]. In 1999, the first multi-part mirror designed to obtain specific properties of the image was presented at RoboCup [10,11].

Many researchers have used several distinct approaches to self-localization in either indoor or outdoor environments, and either using natural or artificial environment landmarks [12]. One currently popular approach is the so-called Markov localization methods $[13,14]$.
An increasing number of teams participating in RoboCup-Soccer middle-size league is approaching the self-localization problem. The proposed solutions are mainly distinguished by the type of sensors used: laser range finders (LRFs), vision-based omni-directional sensors and single frontal camera. The CS-Freiburg and Stuttgart-Cops teams can determine their position with an accuracy of 1 and $5 \mathrm{~cm}$, respectively, using LRFs [15]. However, LRFs require walls surrounding the soccer field to acquire the field border lines and, in a sense, correlate them with the field rectangular shape to determine the team postures. RoboCup's Agilo team [16] proposes a single frontal camera to match a $3 \mathrm{D}$ geometric model of the field with the border lines and goals line segments in the acquired image. Only a partial field view is used in this method. Iocchi and Nardi [17] also use a single frontal camera and match the lines with a field model using the Hough transform. Even though similar to the work on vision-based self-localization described in this paper, their approach considers lines detected locally (again due to a partial field view), rather than a global field view, and requires odometry to remove ambiguities. The robots of the Tübingen team use omni-directional vision for self-localization, but only the distance to the walls is used [18]. Several teams use a vision-based omni-directional catadioptric system similar to the one described here, but only for ball and opposing robots tracking.

Omni-directional vision-based approaches to self-localization have been used already outside RoboCup. One such approach is described in [19], where the authors use a conic mirror to implement a catadioptric vision system that extracts radial straight lines from the surrounding environment, and an extended Kalman filter to integrate the localization data so-obtained by triangulation with odometry.

This paper is organized as follows. In Section 2, the design of the multi-part omni-directional mirror is described. Applications to robotic soccer based on omni-directional catadioptric vision systems, which can use the different parts of the multi-part mirror are introduced in Sections 3 and 4: virtual sensors that locate relevant objects/landmarks in the scene and a self-localization algorithm, respectively. Finally, some conclusions and a description of envisaged future work are drawn in Section 5. 


\section{Designing a multi-part omni-directional mirror}

An accurate design of an omni-directional vision sensor should enable the robot to observe the parts of the scene relevant for the specific application. By analyzing the rules and aims of RoboCup-Soccer middle-size league, it is possible to define a set of requirements for such a vision system.

\subsection{Inferring requirements for the perception system from RoboCup rules}

An omni-directional perception system should be able to detect points of interest (direction and distance) with the accuracy required by the application. The following requirements and applications have been identified in the RoboCup domain:

- When the point of interest is in contact or very near to the robot, a very good accuracy is required for both direction and distance, in order to properly control the robot motion. An example is the control of ball kicking.

- When the point of interest is within a few meters from the robot, a good accuracy is required for both direction and distance. It is very useful that the error affecting distance measurement of scene points in this range to be independent from the points position. An example is self-localization, which based on localization of known points would be eased if such points are observed with the same accuracy.

- When the point is quite far, a good accuracy is required for the direction, less accuracy may be accepted for the distance. An example is moving to the ball: the directional accuracy is required in order to be able to head towards it.

- The last requirement deals with the markers, which allow to distinguish team-mates from opponents. The perception system should be able to observe the markers in the range of distances and heights where they are placed.

In 1999 , a mirror was designed $[10,11]$ only partially matching these requirements. The aims were both to have enough resolution to detect and localize the ball even when observed at the farthest distance, and to include in the image the maximum part of the ball when it is close to the robot body. These requirements could not be matched by any of the classical mirror shapes used till then, and we decided to implement a two-part mirror. The first part was a conical mirror and the second one a spherical apex, sharing a common tangent at the intersection points. The spherical part projected scene points at the ground level up to $1.5 \mathrm{~m}$ from the sensor, thus allowing the angle of the conical part to be steep enough to observe points at a distance up to $6 \mathrm{~m}$ from the sensor (see Fig. 2(b)). The sensor, implemented with a large, low-cost mirror (18.5 cm of diameter) and a low cost camera, was good enough to make it possible the implementation of successful behaviors [11].

Since then, all requirements have been taken into account and the perception system was redesigned. We decided to develop a new design methodology to implement a new set of mirrors based on a comprehensive analysis of the above requirements and satisfying them through an accurate control of the distribution of the image resolution [4].

\subsection{Isometric mirror part}

Vision systems measure the distance between image points in order to estimate the distance between scene points. The relationship between image and scene distances for omni-directional system based on conventional conical mirrors is not linear. Such non-linearity turns into a distortion at the image level (see the outer part of Fig. 2(b)). This distortion grows quickly with the distance from the object to the observer. On one hand, it is quite obvious that the nominal value of the estimates can be easily corrected given the profile function of the mirror. On the other hand, the accuracy of these measurements is corrupted by the joint effect of such distortion and image sampling, without any possibility to compensate for it. The accuracy degradation implied by conventional mirrors conflicts with the requirement of a reasonably limited amount of inaccuracy for any distance measured in the intermediate range. Therefore, one of the objectives of this work was to develop an optical compensation of the above described distortion, working directly on the mirror profile in such a way that the absolute localization error remains limited with respect to the object distance. In other words, the driving idea was to control the distribution of the image resolution on a pixel basis, in order to get the desired accuracy. The analytical set-up for this optical compensation turned out to be very 
similar to previous work [3] (also see [20]), where the aim was to exploit reflective surfaces as computational sensors. This optical compensation results in a constant absolute error in the distance measurement. The transformation between two 2D Euclidean spaces (ground and sensor) performed by such camera-mirror system, keeps angles unchanged and changes lengths by a constant factor. This transformation, being linear, does not change the metric tensor, neglecting the constant. Therefore, we call this kind of mirror isometric because of its capabilities to keep the scene metric, property that does not hold for conventional mirrors.

An even more relevant point driving our design concerns the detection of image features. The proposed design has the effect of keeping constant the image size of the scene features at the ground level, inside the covered range of distances. This makes less likely a detection failure when the feature is far from the observer.

The design problem is modeled by the following differential equation (1), which can be inferred by applying the laws of linear optics (see Fig. 3):

$$
\begin{aligned}
& \frac{\frac{X}{Y}+\frac{2 Y^{\prime}}{1-Y^{\prime 2}}}{1-\frac{X}{Y} \frac{2 Y^{\prime}}{1-Y^{\prime 2}}}=\frac{\eta Y-X^{2}}{X(Y+H)}, \\
& Y(0)=Y_{0}, \quad Y^{\prime}(0)=0,
\end{aligned}
$$

where $Y^{\prime}=\mathrm{d} Y / \mathrm{d} X, \eta=k \lambda, \lambda$ is the focal length, $k$ is the proportionality constant from $X$ to $x, H$ is the pin-hole height from the ground. Differently from $[3,20]$, we developed a geometrical integration of Eq. (1). Our approach is based on a local first-order approximation of the profile, at each point the mirror has been approximated by its tangent space. The resulting profile looks quite similar to the one obtained in [3]. It is convex into its first half, i.e., the part that goes from the axis of symmetry towards the outside of the mirror; then it has an inflection point and finally it gets slightly concave. Establishing point by point the relationship between the mirror profile and the scene is one way to control the distribution of the image resolution. Establishing the amount of image resolution devoted to a single part is an another way to control the distribution of the image resolution.

\subsection{Constant curvature mirror part}

It would be desirable if the above described design approach could cover the whole range of distances required for the RoboCup purposes, but the use of conventional low-cost color cameras does not allow a reliable detection of relevant features in the whole range of distances. Thus, we have designed a second mirror part that satisfies jointly two requirements of the previously mentioned ones. The first of such requirements is the covering of the farthest range of distances. The second is the markers detection and localization. These

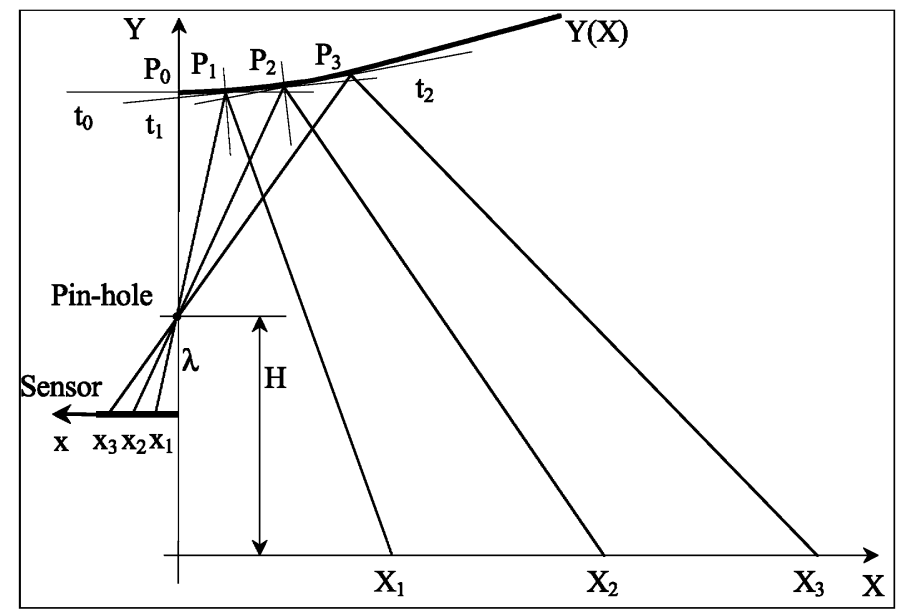

Fig. 3. Sketch for inferring the differential equation generating the isometric part of the mirror $\left(\left(x_{i+1}-x_{i}\right)=k\left(X_{i+1}-X_{i}\right) \forall i\right)$. 


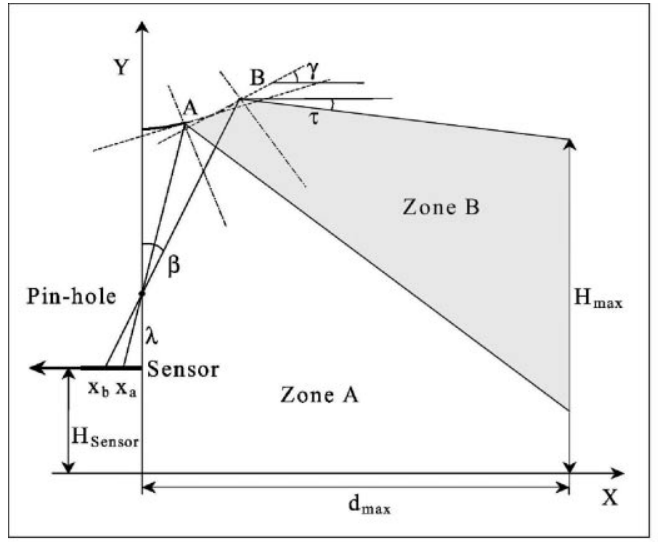

(a)

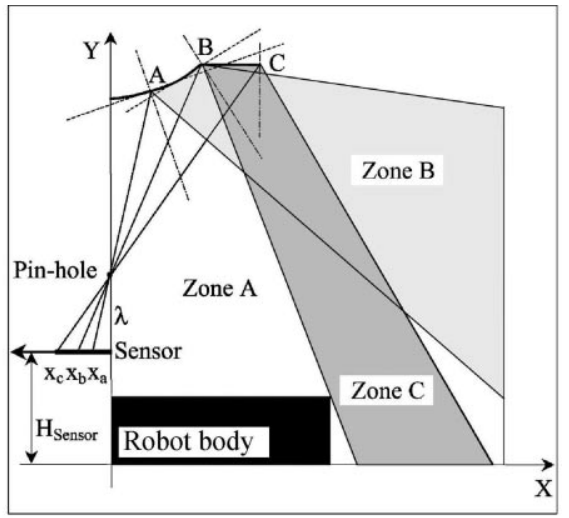

(b)

Fig. 4. (a) Sketch for the design of the constant curvature part of the mirror; (b) sketch for the design of the planar part of the mirror.

two requirements do not imply an accuracy as high as for the other ones. Another aspect of the design of this part is to preserve the continuity between the two portions of the image in order to ease the association of the robot body to its marker, when they are across the two parts. Such image continuity can be guaranteed by imposing the continuity of the tangent at the junction between the isometric and the new part of the mirror (point A in Fig. 4(a)). Another condition comes from fixing point $\mathrm{B}=\left(X_{\mathrm{B}}, Y_{\mathrm{B}}\right)$ and setting the height $H_{\text {max }}$, so that it can be observed at a distance $d_{\text {max }}$. This constraint gives the tangent to the profile in point $\mathrm{B}$

$\tan (\theta)=\frac{H_{\max }-Y_{\mathrm{B}}}{d_{\max }-X_{\mathrm{B}}}, \quad \tan (\beta)=\frac{x_{\mathrm{b}}}{\lambda}$,

$\tan (\gamma)=\tan \left(\frac{\beta+\theta-\pi}{2}\right)$,

where $\lambda$ is the focal length.

Because there is no other constraint, this portion of the mirror can be designed, e.g., by imposing a constant variation of the tangent between the two endpoints. Hence, the name "constant curvature" given to this part of the mirror. The mirror will cover completely the highest part of the scene (Zone B). On the other hand, when the robots are quite near, they will be observed by the first part of the mirror (Zone A).

\subsection{Planar mirror part}

The so far designed mirror does not satisfy the requirement concerning the nearer range of distances. Due to the robot occlusion (see Fig. 4b), it is not possible to observe the scene immediately close to the robot. The relatively small image of a feature, when very near and imaged in the isometric part, results in a less than required accuracy, while the highest should be obtained in the very close range. To satisfy the requirement, a third part of the mirror has been introduced. This part should be the outmost to suffer the least occlusion from the robot body. The simplest solution to this design problem is a planar mirror lying on a plane perpendicular to the rotational axis. The height of this part has to be as low as possible, with respect to the camera, in order to give the largest images of the features. At the same time, this part should not be on the line sight of others. Hence, the choice has been to have a planar mirror at the same height of the last point of the constant curvature part of the mirror (point $\mathrm{B}$ ). The point $\mathrm{C}$ is set as follows:

$\frac{X_{\mathrm{C}}}{Y_{\mathrm{C}}-\lambda-H_{\text {sensor }}}=\frac{x_{\mathrm{c}}}{\lambda}, \quad Y_{\mathrm{C}}=Y_{\mathrm{B}}$,

where $H_{\text {sensor }}$ is the height of sensor plane and $\lambda$ is the focal length. The ball image produced by this part is large enough to allow a reliable detection and an accurate localization. 


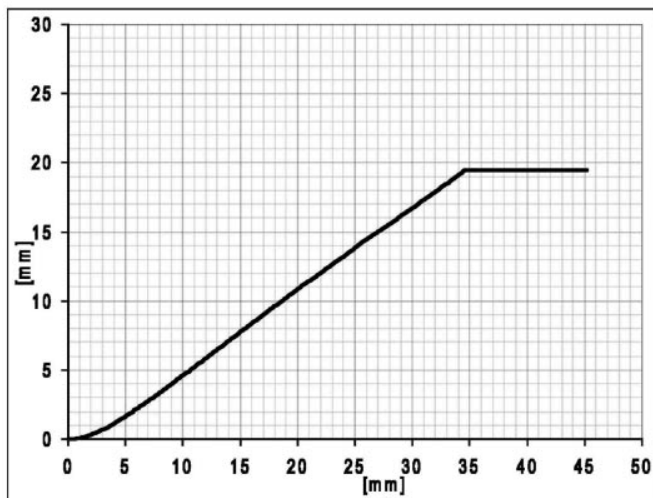

(a)

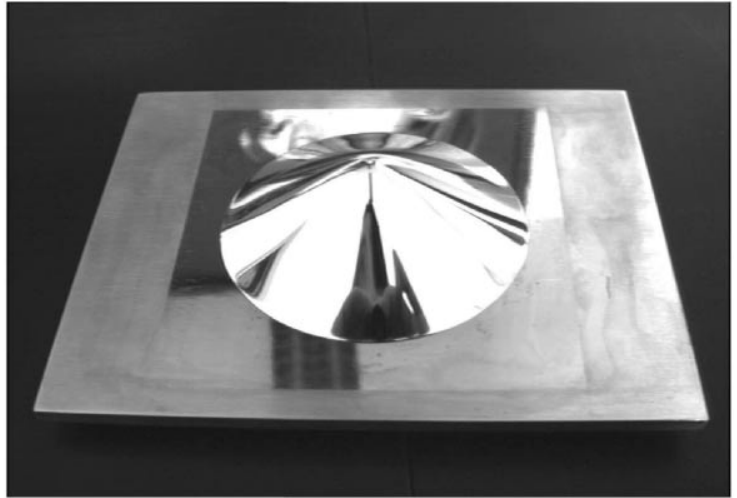

(b)

Fig. 5. (a) Profile of the overall mirror; (b) the last mirror prototype.

\subsection{The resulting mirror}

The mirror profile resulting from the above described design is shown in Fig. 5(a). It enables the system to observe up to $6 \mathrm{~m}$ far away without image distortion at the ground level; thanks to the constant curvature part it can observe up to the maximum height, $0.6 \mathrm{~m}$, at the maximum distance in the ground $(11.2 \mathrm{~m})$. Its outer part allows the observation of objects from 0.39 to $0.51 \mathrm{~m}$. The last prototype of the mirror is depicted in Fig. 5(b), an image obtained by this mirror and a very low-cost camera is shown in Fig. 6. You may notice that such image have been collected after a rough mechanical set-up. This activity should have aligned optical and mirror axis, put the mirror at the designed distance from the pin-hole, etc. It is extremely likely that some defect is still present on the image.

\section{Virtual image sensors for robotic soccer}

Many visual features are important in the RoboCupSoccer domain. A set of virtual image sensors ${ }^{5}$ was designed to extract a crucial subset of those features for middle-size league robots, and handle the necessary actions, namely:

\footnotetext{
${ }^{5}$ A virtual image sensor extracts features from a (sub)image, such as the centroid or whether an object is present or not. Different virtual sensors operate over the image provided by just one transducer: the CCD camera plus the video acquisition board.
}

- other robots and walls, for obstacle avoidance;

- goals;

- far ball, to move towards it;

- near ball, to kick it;

- catadioptric system calibration.

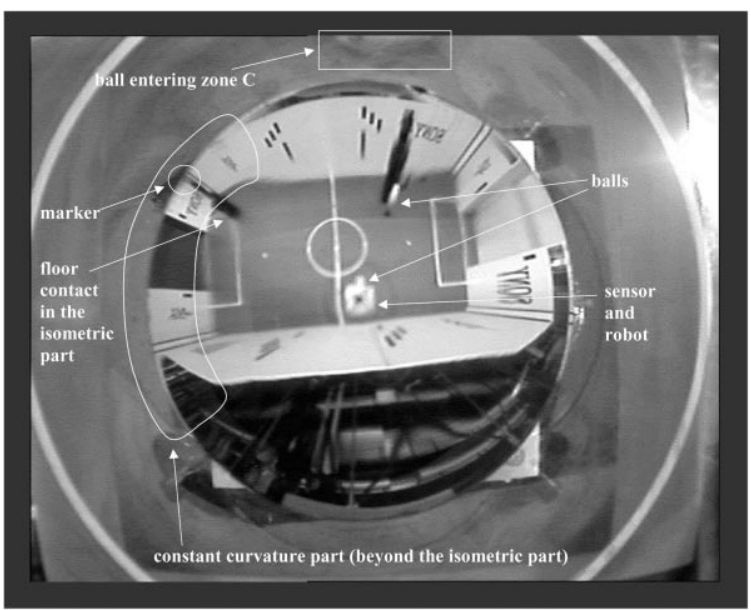

Fig. 6. Image taken with the robot near to the center of the ground (Melbourne, 31 August 2000, field B of the initial tournament). Notice the effect of the isometric optical compensation, which lasts up to $6 \mathrm{~m}$; in the constant curvature part it is possible to detect a goal and the marker. However, their distances from the observer, thanks to the continuity with the first part, can be measured at the ground contact point with the limited error provided by the isometric design. Notice also the dimension of the farther ball, which is even larger than that when nearby because of the isometric property holding at the floor level only. There was no marker on the robot besides the farther ball. 
Those virtual sensors can be used with any catadioptric vision system, particularly the one described in the precedent section. This section goes through their implementation details for a parabolic mirror that was built to test only the virtual sensors.

\subsection{The importance of colors}

After capturing an image, what can be done with it in order to instruct a robot to play soccer? An important step is the reliable extraction of visual features from the image, corresponding to relevant objects on the field.

First, the objects must be recognized. These are the two goals, the ball, the surrounding walls, the other robots and their markers. All those objects are recognized by their known colors. Eight different colors are used:

- the ball is red;

- the playing field is green;

- one goal is blue;

- the other goal is yellow;

- the surrounding walls are white (including some letters and symbols in black);

- the robots are predominantly black;

- one team color is magenta;

- the other team color is cyan.

These eight colors correspond precisely to the eight vertices of the RGB cube [21].

Color segmentation is obviously an important problem for RoboCup-Soccer playing robots. The image processing system must not only correctly discriminate the eight significant colors, but also avoid the identification of objects external to the game as relevant ones. This is a critical issue since many people walks around the playing field wearing colorful T-shirt, and sometimes other red balls are left nearby the field.

\subsection{Image formats and color segmentation}

Different cameras provide images in different formats. The most usual ones are RGB and YUV. Due to its video characteristics, YUV is the most suitable color space for color segmentation. Its main advantages can be described as follows:
- the signal is separated (to analyze shape, we do not need color, but just luminance);

- it is very much light independent;

- it is fast, since no hardware conversion is required;

- lookup tables are 2D, and thus they are easy to access and require less storage space than "true" 3D color spaces;

- allows flexible conversions to RGB for display;

- many cameras output their image in the YUV format.

The main disadvantages are the following:

- it needs to be converted to RGB to be displayed on a computer screen;

- it is a format most suitable for video rather than for still images.

\subsection{Sensor readings from an image system}

Most sensorial information required in RoboCupSoccer can be extracted by a vision system. Our approach was to define image windows where certain attributes are expected to be found. An example can be seen in Fig. 7, for a parabolic mirror. Notice that the image windows must be changed according to the particular mirror profile used and/or mirror assembly on the robot. This system starts by applying a filter to every image pixel. All colors are segmented onto the eight possible and acceptable options.

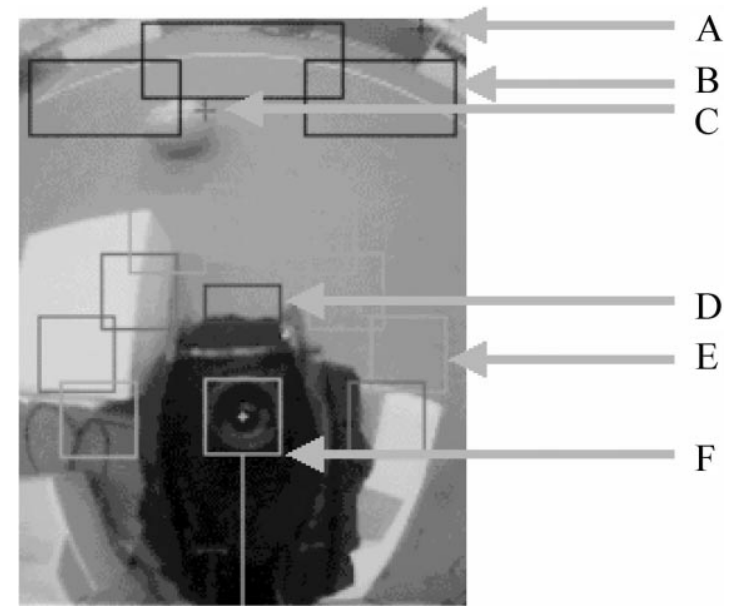

Fig. 7. Captured image with superimposed defined windows for virtual sensors. 
The main virtual sensors used in this system are designated as Obstacle Avoidance, Goal Detection, Ball Detection, Eminent Kick and Catadioptric System Calibration. We shall now describe each of them in detail:

- Obstacle Avoidance. The nine squares around the camera are used as virtual bumpers (window $\mathrm{E}$ in Fig. 7). The amount of black and white inside each square is calculated, and should it be over a certain predefined value, an obstacle is detected, forcing the motion controller to move the robot away from the obstacle. The "bumpers" are numbered from 1 to 9 , starting on the left side. In the image shown, bumpers 2, 3 and 9 are darker which means they are flagged showing evidence of an obstacle detected.

- Goal Detection. By finding the maximum value of blue (window A in Fig. 7) in the image and applying a threshold, the blue goal can be found. A similar technique is used for the yellow goal. In order to avoid noise from outside the field, which could be confused with the actual blue (yellow) goal, this maximum must be inside the top three rectangles on the image (window B in Fig. 7).

- Ball Detection. The maximum value of red (window $\mathrm{C}$ in Fig. 7) represents the ball. The red color is the easiest to track and the one with least interference, since the ball has a very unique and bright color. Due to its motion, the ball can be seen anywhere on the image, and so window $\mathrm{C}$ can be located.

- Eminent Kick. The robot should not activate the kicking device when the ball is not ready to be kicked, to save energy and avoid hurting its opponents. Therefore, the kicking device is activated only when the ball (red cross represented by letter $\mathrm{C}$ in Fig. 7) is inside the red rectangle (letter D on the same image). This also means that the robot will kick the ball only when the ball is touching the robot.

- Catadioptric System Calibration. Should, for some reason, the mirror and/or the camera composing the catadioptric vision system be moved from its position, the robot will not find the relevant objects in the correct image windows. Therefore, for easy calibration of the catadioptric system, the camera lens must be placed inside the square given by letter F in Fig. 7.
Many other virtual sensors can be created. However, the number of sensors is critical for system performance and therefore their number must be limited, otherwise, the number of frames processed per second will substantially decrease.

\section{Omni-directional vision-based self-localization}

The navigation system is one of the most important sub-system of a mobile robot. In many applications, especially those concerning well-structured indoor environments, one important feature of the navigation system concerns the ability of the robot to self-localize, i.e., to autonomously determine its position and orientation (posture). Once a robot knows its posture, it is capable of following a pre-planned virtual path or of smoothly stabilizing its posture. If the robot is part of a cooperative multi-robot team, it can also exchange the posture information with its team-mates, so that appropriate relational and organizational behaviors are established. In robotic soccer, these are crucial issues. If a robot knows its posture, it can move towards a desired posture (e.g., facing the goal with the ball in between). It can also know its team-mate postures and prepare a pass, or evaluate the game state from the team locations [22].

In this section, we describe a self-localization algorithm based on the isometric part of the multi-part mirror of the catadioptric vision system described in Section 2. The algorithm determines the posture of a middle-size league robot, with respect to a given coordinate system, from the observation of natural landmarks of the soccer field, such as the field lines and goals, as well as its correlation, in the Hough transform space, with a geometric field model. Even though the intersection between the field and the walls is also currently used, the wall replacement by the corresponding field lines would not change the algorithm. The algorithm is a particular implementation of a general method applicable to other well-structured environments, and was first introduced in [23].

\subsection{Method description}

Even though the self-localization algorithm was designed motivated by its application to robotic soccer, it can be described in general terms, and applied to 
other well-structured environments, with the assumption that the robot moves on flat surfaces and straight lines can be identified and used as descriptive features of those environments. An important requirement is that the algorithm should be robust to image noise. Given an image acquired from the isometric part of the catadioptric system, the basic steps of the algorithm are the following:

1. Build a set $T$ of transition pixels, corresponding to image pixel representatives of environment straight lines (e.g., intersection between corridor walls and ground, obtained by an edge detector).

2. For all transition pixels $p^{\mathrm{t}} \in T$, compute the Hough transform [21] using the normal representation of a line

$\rho=x_{i}^{\mathrm{t}} \cos (\phi)+y_{i}^{\mathrm{t}} \sin (\phi)$,

where $\left(x_{i}^{\mathrm{t}}, y_{i}^{\mathrm{t}}\right)$ are the image coordinates of $p^{\mathrm{t}}$ and $\rho, \phi$ the line parameters.

3. Pick the $q$ straight lines $\left(\rho_{1}, \phi_{1}\right), \ldots,\left(\rho_{q}, \phi_{q}\right)$ corresponding to the top $q$ accumulator cells resulting from the Hough transform described in the previous step.

4. For all pairs $\left\{\left(\rho_{j}, \phi_{j}\right),\left(\rho_{k}, \phi_{k}\right), j, k=1, \ldots, q\right.$, $j \neq k\}$ made out of the $q$ straight lines in the previous step, compute

$$
\begin{gathered}
\Delta \phi=\left|\phi_{j}-\phi_{k}\right|, \\
\Delta \rho=\left|\rho_{j}-\rho_{k}\right| .
\end{gathered}
$$

Note that a small $\Delta \phi$ denotes almost parallel straight lines, while $\Delta \rho$ the distance between two parallel lines.

5. Classify, in the $[0,100]$ range, the $\Delta \phi \mathrm{s}$ and $\Delta \rho \mathrm{s}$ determined in the previous step, for its relevance (function Rel(.)) using a priori knowledge of the geometric characteristics of the environment (e.g., in a building corridor of width $d$, only $\Delta \phi \simeq 0$, $\Delta \phi \simeq 180$ and $\Delta \rho \simeq d$ should get high grades). For each pair of straight lines, assign a grade in the $[0,200]$ range to the pair, by adding up $\operatorname{Rel}(\Delta \phi)$ and $\operatorname{Rel}(\Delta \rho)$.

6. Pick up the most relevant pair of straight lines (i.e., the pair of largest $\operatorname{Rel}(\Delta \phi)+\operatorname{Rel}(\Delta \rho)$ in the previous step), and use it to extract some relevant feature regarding environment localization (e.g., the orientation $\theta$ of the robot w.r.t. the corridor walls, represented by the most relevant pair of parallel straight lines, in the example above).

7. Use the relevant feature from the previous step to proceed. For instance, assuming $\theta$ in the corridor example is such a feature, it is used to select columns from the accumulator cells matrix referred in Step 3. The idea is to correlate a number of actual straight lines, found in the image, sharing the same descriptive parameter (e.g., the angle $\phi$ corresponding to $\theta$ ) with the expected straight lines obtained from an environment model (e.g., the building layout). To attain this, up to $n \rho$ values from the accumulator matrix column corresponding to $\phi$ are picked up, corresponding up to $n$ straight lines found in the image. To handle uncertainty in $\phi$, an even better solution is to pick up not only one column but a few columns surrounding the accumulator matrix column corresponding to $\phi$, using the top $n \rho$ values from those columns. Concatenate all these Hough space points in an array and call it $\hat{\rho}_{\phi}$.

8. Create an array $\rho_{\phi}$ similar to $\hat{\rho}_{\phi}$, but obtained from a geometric model of the environment. Actually, $\rho_{\phi}$ measures distances of environment straight lines to the origin of the world reference frame. Correlate $\rho_{\phi}$ and $\hat{\rho}_{\phi}$ by shifting one array over the other, and incrementing a counter for each matching $\left(\rho_{\phi}, \hat{\rho}_{\phi}\right)$ pair. The maximum of the correlation corresponds to the best match between up to $n$ straight lines in the image and the $n$ known environment straight lines. From this result and similar results obtained for other straight lines non-parallel to them (determined by the same procedure for different $\theta \mathrm{s}$ ), the image coordinates of environment feature points, whose location in the world reference frame is known, are determined and used to determine the robot position w.r.t. that frame, by a suitable transformation from image to world coordinates.

\subsection{Application to robotic soccer}

The self-localization of a middle-size league soccer robot, using the method described in the previous section, takes advantage of the soccer field geometry and of the different colors used for the field (green), the surrounding walls and the field lines (white). The field is a $9 \mathrm{~m} \times 4.5 \mathrm{~m}$ flat rectangle that can be almost fully observed by the robot catadioptric system from most field locations. 
The self-localization algorithm was implemented based on the isometric part of the catadioptric system mirror.

\subsection{Geometric field model}

The bird's eye view of the soccer field, shown schematically in Fig. 9(a), shows six horizontal and seven vertical straight lines (considering interrupted lines as only one line). In this work, all horizontal lines and five of the vertical lines (excluding those corresponding to the back of the goals) were considered. Excluded lines were chosen because they are often occluded by the goalkeeper robots. All the distances between lines are known from RoboCup rules. Changes in the dimensions are parameterized in a table. The model reference frame is located at the bottom left of the model image.

\subsection{Orientation determination}

Steps 1-6 of the algorithm described in Section 4.1 are followed to determine the initial robot orientation estimate (with a $\pm 90^{\circ}$ or $0 / 180^{\circ}$ uncertainty, to be solved later). The set $T$ of transition pixels is obtained by determining the white-to-green and green-to-white image transitions over 36 circles centered with the robot, shown in Fig. 8. The number of circles was determined based on a trade-off between accuracy and CPU time.

The Hough transform is then applied to the pixels in $T$ - a variable number from image to image $-\mathrm{de}$ -

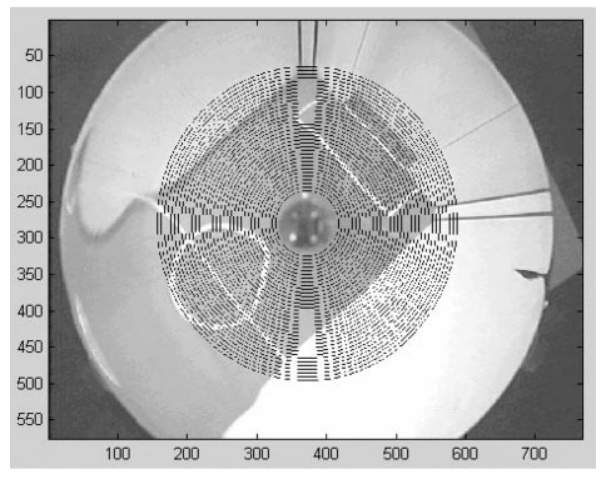

Fig. 8. Image obtained with a preliminary prototype of the isometric part of the catadioptric system mirror - notice the distortion on the outer part - showing the 36 circles used to determine transition pixels. pending on the number and length of observed lines. In Step 3, $q=6$ is used, based on experimental analysis of the trade-off between CPU time and accuracy. The relevance functions for $\Delta \phi$ and $\Delta \rho$, used in Steps 5 and 6, are plotted in Fig. 9(b) and (c). The latter reflects a priori knowledge of the environment by its use of the known distance between relevant field lines that can be observed by the catadioptric system in one image.

The accumulator cells of the Hough transform in Step 2 are obtained by incrementing $\phi$ from $0^{\circ}$ to $180^{\circ}$ in $0.5^{\circ}$ steps, leading to a line slope resolution in the image of $\tan 0.5^{\circ} \cdot \rho$ is incremented from 125 to 968 in steps of 1 pixel, corresponding to an actual field resolution of $6.95 \mathrm{~mm} .{ }^{6}$ The $\pm 90^{\circ}$ or $180^{\circ}$ ambiguity referred above results from the absence of information on which field lines lead to the most relevant pair. This information is obtained in Steps 7 and 8.

\subsection{Position determination}

The final step in the self-localization process consists of determining the robot position coordinates in the soccer field. This is done together with the disambiguation of the relevant feature $\theta$ determined in Steps 1-6 of the self-localization method, by creating not only the $\rho_{\phi}$ and $\hat{\rho}_{\phi}$ arrays referred in Steps 7 and 8 , but also their "orthogonal" arrays $\rho_{\phi+90}$ and $\hat{\rho}_{\phi+90}$. The correlation in Step 8 is made between all four possible pairs $\left(\rho_{\phi+90}, \hat{\rho}_{\phi+90}\right),\left(\rho_{\phi+90}, \hat{\rho}_{\phi}\right),\left(\rho_{\phi}, \hat{\rho}_{\phi+90}\right)$ and $\left(\rho_{\phi}, \hat{\rho}_{\phi}\right)$ with $n=6$ (the maximum number of field lines that can be found in the image). The maximum of the four correlation maxima occurs for the array pair representing the best match between image and actual field lines. The array immediately identifies whether $\theta \pm 90^{\circ}$ or $\theta=0^{\circ} \vee 180^{\circ}$ is the robot orientation. A companion array pair exists for each best pair. The two pairs uniquely identify two (approximately) orthogonal field lines, by checking the array positions where the maximum occurred (vertical field lines are numbered $1, \ldots, 5$ from left to right and horizontal lines are numbered $1, \ldots, 6$ from top to bottom). The intersection of the two lines is a reference point, whose coordinates are known in the world reference frame, from the field model.

\footnotetext{
${ }^{6}$ The relation between $\rho$ values and the actual field resolution is given by the scale factor $k$ between field and image coordinates (see Section 2.2).
} 


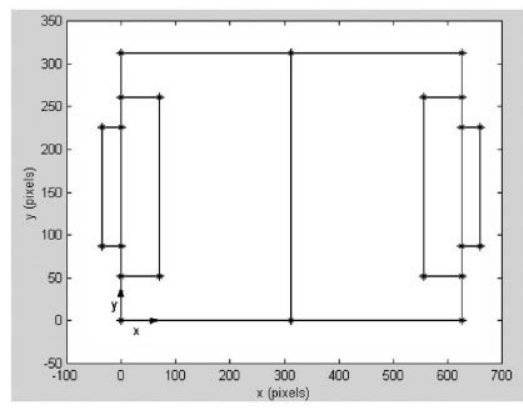

(a)

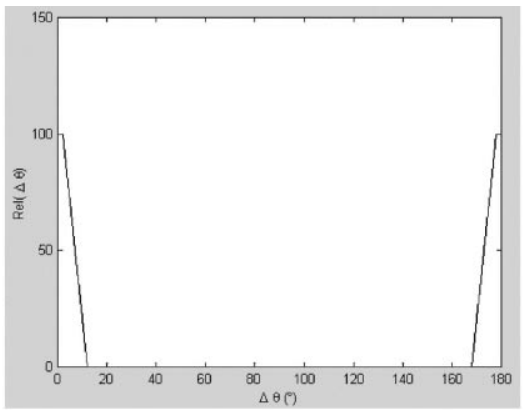

(b)

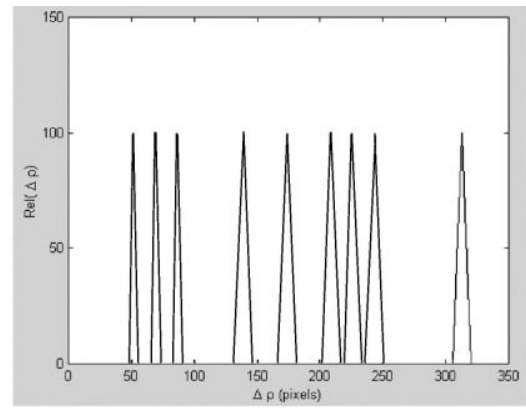

(c)

Fig. 9. (a) Soccer field model as seen in a bird's eye view image (coordinates in pixels). Also shown are the relevance functions for (b) $\Delta \phi$ and (c) $\Delta \rho$.

The explanation above is summarized in the following table (the best and companion pairs positions can be exchanged)

\begin{tabular}{lll}
\hline Best pair & Companion pair & $\theta$ \\
\hline$\left(\rho_{\phi}, \hat{\rho}_{\phi}\right)$ & $\left(\rho_{\phi+90}, \hat{\rho}_{\phi+90}\right)$ & $\phi \pm 90^{\circ}$ \\
$\left(\rho_{\phi}, \hat{\rho}_{\phi+90}\right)$ & $\left(\rho_{\phi+90}, \hat{\rho}_{\phi}\right)$ & $\phi \vee \phi \pm 180^{\circ}$ \\
\hline
\end{tabular}

The robot position is computed from a rotation of $\theta$ (one of the possible values is used, with no special criterion), followed by a translation that expresses the center of the image (i.e., the robot position in image coordinates) in the model reference frame, and another translation plus a scale factor $f$ to express it in world coordinates. The world reference frame is located in the middle of the soccer field, with the $x$-axis pointing towards the blue goal and the $y$-axis is such that a $3 \mathrm{D}$ coordinate frame would have $z$-axis pointing upwards. The orientation $\theta$ is measured from $x$ to the straight line passing through the robot center and the center of the robot front. The scale factor $f$ depends on the geometry of the catadioptric system and can be calibrated experimentally. This transformation can be expressed by the following equation, using homogeneous coordinates:

$$
\begin{aligned}
{\left[\begin{array}{c}
x_{\mathrm{f}}^{\mathrm{r}} \\
y_{\mathrm{f}}^{\mathrm{r}} \\
1
\end{array}\right]=} & {\left[\begin{array}{ccc}
\cos \theta & \sin \theta & x_{\mathrm{i}}^{\mathrm{ref}}+x_{\mathrm{m}}^{\mathrm{ref}} \\
-\sin \theta & \cos \theta & y_{\mathrm{i}}^{\mathrm{ref}}+y_{\mathrm{m}}^{\mathrm{ref}} \\
0 & 0 & 1
\end{array}\right]\left[\begin{array}{c}
x_{\mathrm{i}}^{\mathrm{r}} \\
y_{\mathrm{i}}^{\mathrm{r}} \\
1
\end{array}\right] } \\
& -\left[\begin{array}{c}
450 \\
225 \\
0
\end{array}\right] f,
\end{aligned}
$$

where the subscripts $\mathrm{i}, \mathrm{m}$ and $\mathrm{f}$ stand for the image, field model and actual field reference frames, and the superscripts ref and $\mathrm{r}$ stand for the reference point and the robot, respectively.

A further validation and disambiguation of the robot posture is required, since, when only two parallel lines are used to determine the position, and due to field symmetry, the robot side of the field is unknown, as well as its orientation. To solve this problem, two tests are made. First, the algorithm checks whether the robot position is not outside the field. The second test consists of using the current estimated posture to seek the nearest goal in the image.

This is achieved by selecting $m$ points located inside one of the goals (blue or yellow) in the actual field and applying to each of those points of the coordinates $\left(x_{\mathrm{f}}^{\mathrm{g}}, y_{\mathrm{f}}^{\mathrm{g}}\right)$ the inverse transform of (7).

Should the majority of the corresponding pixels in the image have the same color of the field pixels, $\theta=$ $0^{\circ}$ and the estimated position is validated. Should they have the color of the opposing goal, $\theta=180^{\circ}$ and the symmetrical coordinates of the current position estimated must be used for the robot position. When the majority of image pixels is green, the top maximum of the correlation process is removed and the whole process re-started using the second maximum, and if needed, the third one and so on until the actual posture is determined.

\subsection{Experimental results}

The described self-localization algorithm has been implemented in $\mathrm{C}$. The method was applied to a set of 

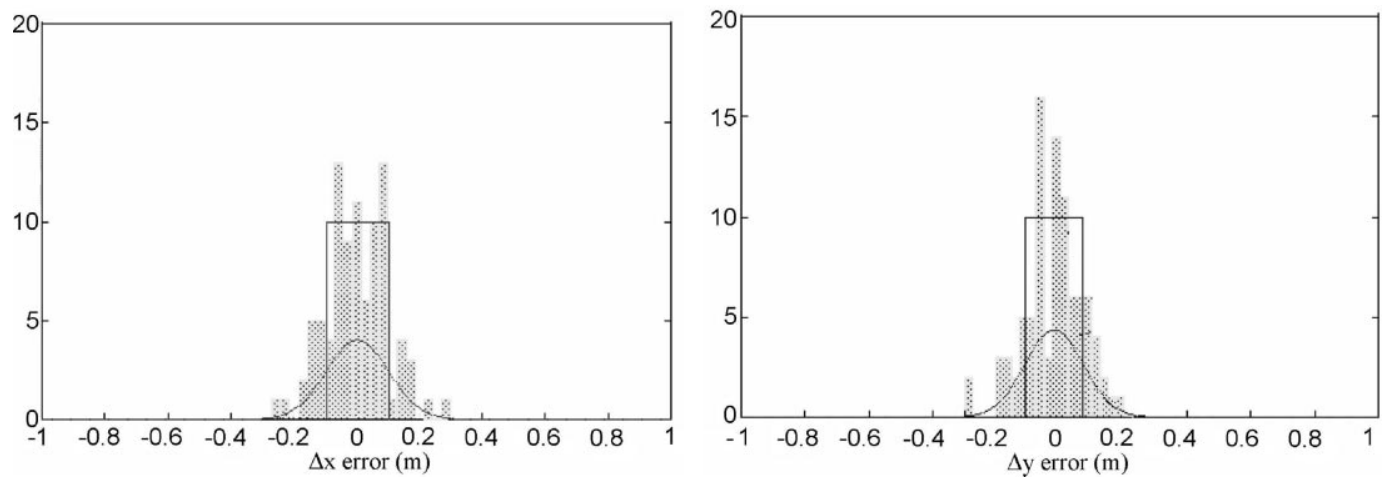

Fig. 10. Position error histogram.

90 images obtained by a catadioptric system mounted on a Super Scout II robot. The images were taken at different field spots with several images taken at each spot, and were processed in about $0.5 \mathrm{~s}$ each in a Pentium $233 \mathrm{MHz}$ with $64 \mathrm{MB}$ of RAM, the Super Scout II on board computer. The results from the 90 experiments give an average accuracy $\mu$ of $3.2 \mathrm{~mm}$ for the $x$-coordinate, $-18 \mathrm{~mm}$ for the $y$-coordinate and $0.22^{\circ}$ for $\theta$, with standard deviations of $100,92 \mathrm{~mm}$ and $1.8^{\circ}$, respectively.

In Fig. 10, the histogram of the accuracy, for the $x$ - and $y$-coordinates, is shown, as well as an adjusted Gaussian function. The rectangle on the plot contains all the accuracies within one standard deviation from $\mu$, i.e., $68.2 \%$ of the postures obtained have an accuracy of less than or equal to $10 \mathrm{~cm}$ in $x$ and $9 \mathrm{~cm}$ in $y$.

The accuracy was determined as the difference between the estimated values and the ones measured on the field, using pre-defined spots whose location is well known (e.g., the corner of the goal area). The precision (i.e., the difference between the measured value and the measurements average value for the same location) results are similar, and visual inspection made the average values seem trustable.

Fig. 11 shows an example of an image to be processed. The lines represented are the possible lines of the field. In this case, the best pair was $\left(\rho_{\phi}, \hat{\rho}_{\phi+90}\right)$ and posture was estimated with an error of $\Delta x=+1 \mathrm{~cm}$, $\Delta y=+1 \mathrm{~cm}$ and $\Delta \theta=+1^{\circ}$. Note that, in this test,
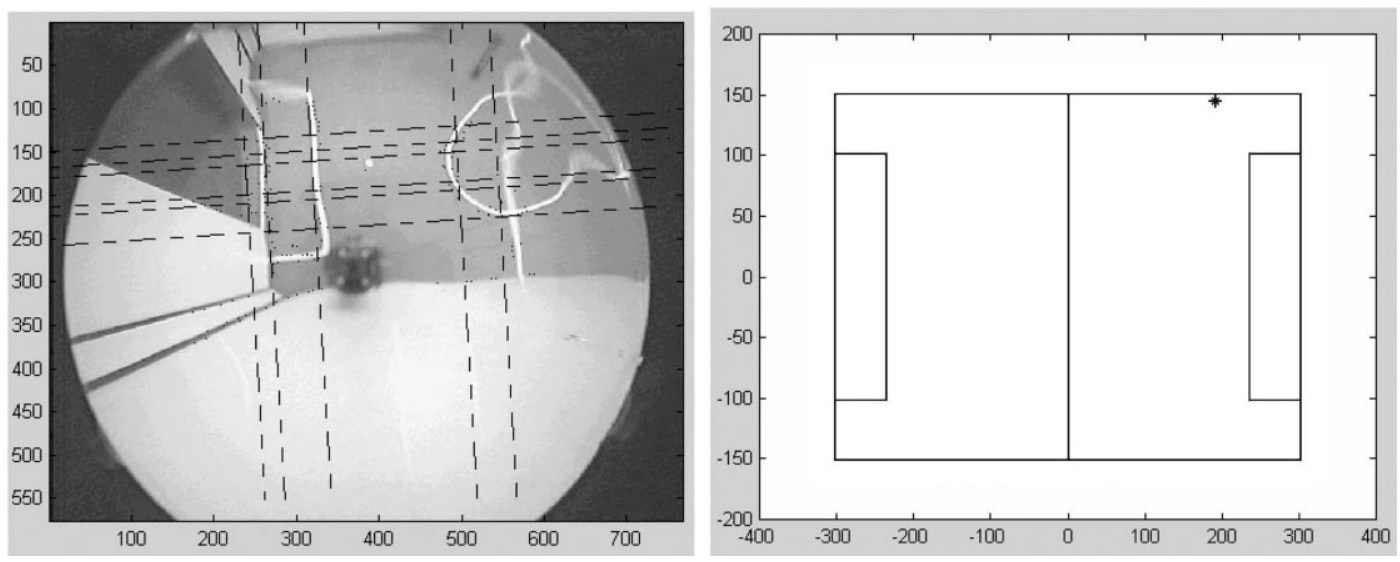

Fig. 11. Test image results. 
the robot is close to one of the field walls making harder the posture determination process, because the other wall is not seen, and a relevant parallel line cannot be found by the algorithm.

\section{Conclusions}

This paper has shown the potential of omnidirectional catadioptric systems for comprehensive solutions for mobile robots moving within structured environments, ranging from the extraction of relevant image features to self-localization. Moreover, this paper introduces the design of a multi-part mirror which can be used, by controlling the distribution of image resolution onto the scene, to tackle all the requirements with the same device.

Further steps towards a more refined usage of the information provided by omni-directional vision systems, as described here, include:

- Endowing many team-mates with such a system, so that they can share information on all team-mate postures through communications, enabling the display of teamwork behaviors.

- Sharing also the information on the position of other relevant objects (e.g., the ball, the opponent robots), observed by each self-localized robot, so that a more accurate world model can be built and shared by all team-mates.

\section{References}

[1] S. Nayar, Catadioptric vision, in: Proceedings of the Seventh International Symposium on Intelligent Robotic Systems, SIRS'99, Coimbra, Portugal, 1999, pp. 1-11.

[2] F. Ribeiro, C. Machado, S. Sampaio, B. Martins, Image processing applied to a robotic football team, in: G. Adorni, W. van der Hoek (Eds.), Proceedings of the EuRoboCup 2000 Workshop, Amsterdam, 2000, on a CD-ROM.

[3] R.A. Hicks, R. Bajcsy, Reflective surfaces as computational sensors, in: IEEE Workshop on Perception for Mobile Agents - Proceedings of the 1999 IEEE Conference on Computer Vision and Pattern Recognition, CVPR'99, IEEE Computer Press, Piscataway, NJ, 1999.

[4] F.M. Marchese, D.G. Sorrenti, Omni-directional vision with a multi-part mirror, in: P. Stone, T. Balch, G. Kraetzschmar (Eds.), RoboCup 2000 - Robot Soccer World Cup IV, Springer, Berlin, 2001.
[5] Y. Yagi, S. Kawato, S. Tsuji, Real-time omni-directional image sensor (copis) for vision-guided navigation, IEEE Transactions on Robotics and Automation 10 (1) (1994) 1122.

[6] E. Mouaddib, C. Pegard, Localization using omnidirectional vision, in: Proceedings of ICAR-95, IEEE Computer Press, Piscataway, NJ, 1995, pp. 133-138.

[7] J.B. Gregersen, A system for $360^{\circ}$ vision in robot soccer, Ph.D. Thesis, RMIT, Melbourne, Australia, 1998.

[8] S. Suzuki, T. Katoh, M. Asada, An application of vision-based learning for a real robot in robocup - Learning of goal keeping behavior for a mobile robot with omni-directional vision and embedded servoing, in: M. Asada, H. Kitano (Eds.), RoboCup 98 - Robot Soccer World Cup II, Springer, Berlin, 1999, pp. 467-474.

[9] D. Nardi, G. Clemente, E. Pagello, ART Azzurra Robot Team, in: M. Asada, H. Kitano (Eds.), RoboCup'98: Robot Soccer World Cup II, Springer, Berlin, 1999, pp. 467474.

[10] A. Bonarini, P. Aliverti, M. Lucioni, An omni-directional sensor for fast tracking for mobile robots, IEEE Transactions on Instrumentation and Measurements 49 (3) (2000) 509 512.

[11] A. Bonarini, The body, the mind or the eye, first? in: M. Veloso, E. Pagello, H. Kitano (Eds.), RoboCup'99 - Robot Soccer World Cup III, Springer, Berlin, 2000, pp. 210-219.

[12] J. Borenstein, H. Everett, L. Feng, Where am I? - Sensors and methods for mobile robot positioning, Technical Report, University of Michigan, Ann Arbor, MI, 1996.

[13] S. Enderle, M. Ritter, D. Fox, S. Sablatnog, G. Kraetzschmar, G. Palm, Vision-based localization in RoboCup environments, in: P. Stone, T. Balch, G. Kraetzschmar (Eds.), RoboCup 2000 - Robot Soccer World Cup IV, Springer, Berlin, 2001.

[14] D. Fox, W. Burgard, S. Thrun, Markov localization for mobile robots in dynamic environments, Journal of Artificial Intelligence Research 11 (1999) 391-427.

[15] P. Stone, T. Balch, G. Kraetzschmar (Eds.), RoboCup 2000 - Robot Soccer World Cup IV, Lecture Notes in Computer Science, Springer, Berlin, 2001.

[16] R. Hanek, T. Schmitt, M. Klupsch, S. Buck, From multiple images to a consistent view, in: P. Stone, T. Balch, G. Kraetzschmar (Eds.), RoboCup 2000 - Robot Soccer World Cup IV, Springer, Berlin, 2001.

[17] L. Iocchi, D. Nardi, Self-localization in the robocup environment, in: Proceedings of 16th IJCAI'99, The Third International Workshop on RoboCup, Stockholm, Sweden, 1999, pp. 116-120.

[18] M. Plagge, A. Zell, Vision-based goalkeeper localization, in: G. Adorni, W. van der Hoek (Eds.), Proceedings of the EuRoboCup 2000 Workshop, Amsterdam, 2000, on a CD-ROM.

[19] L. Delahoche, C. Pégard, B. Marhic, P. Vasseur, A navigation system based on an omni-directional vision sensor, in: Proceedings of the IEEE International Conference on Intelligent Robots and Systems, 1997, pp. 718-724. 
[20] R.A. Hicks, R. Bajcsy, Catadioptric sensors that approximate wide-angle perspective projections, in: IEEE Conference on Computer Vision and Pattern Recognition (CVPR'00), IEEE Computer Press, Piscataway, NJ, 2000.

[21] R. Gonzalez, R. Woods, Digital Image Processing, Addison-Wesley, Reading, MA, 1992.

[22] R. Ventura, P. Aparício, P. Lima, C. Pinto-Ferreira, Isocrob - Intelligent Society of Robots, in: RoboCup-99 Team Descriptions, Middle Size Robots League, 1999, pp. 150159.

[23] C. Marques, P. Lima, A localization method for a soccer robot using a vision-based omni-directional sensor, in: P. Stone, T. Balch, G. Kraetzschmar (Eds.), RoboCup 2000 - Robot Soccer World Cup IV, Springer, Berlin, 2001.

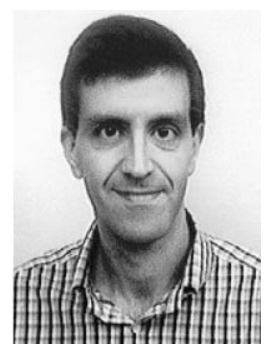

Pedro Lima got his Licenciatura (5 years) and M.Sc. degrees in 1984 and 1989, respectively, in Electrical and Computer Engineering, both from the Instituto Superior Técnico (IST), Lisbon Technical University, Portugal, and his Ph.D. (1994) in Electrical Engineering from the Rensselaer Polytechnic Institute, Troy, NY. Since 1994, he is an Assistant Professor at IST. $\mathrm{He}$ is also a member of the Institute for Systems and Robotics, a Portuguese private research institution. $\mathrm{He}$ is the member of the editorial board of the International Journal of Intelligent Control and Systems, as well as of the editorial board of the Portuguese magazine Robótica, and a member of the Executive Committee of the RoboCup Federation, where he also serves as Co-chair of the middle-size league. In 2001, Pedro Lima is the Chair of the RoboCup-2001 middle-size league, in Seattle. His current research interests include hybrid systems, discrete event systems, reinforcement learning and cooperative robotics. $\mathrm{He}$ is the co-author of a book.

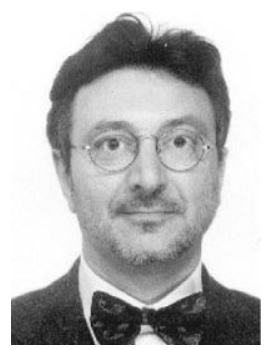

Andrea Bonarini was born in Milan in 1957. He received a Laurea (Master of Technology) in Electronics Engineering in 1984 and a Ph.D. in Computer Science in 1989, both from the Politecnico di Milano, Italy. He is an Associate Professor at the Department of Electronics and Computer Engineering of the Politecnico di Milano. He is a member of the Politecnico di Milano Artificial Intelligence and Robotics (PM-AI\&R Project) since 1984. He is a founding member of the $\mathrm{AI} * \mathrm{IA}$ (the Italian Association for Artificial Intelligence), and among the founders of the IEEE-NN Council Italian RIG. He is coordinating the PM-AI\&R Lab, where he has devel- oped several autonomous mobile robots. He is also participating to the RoboCup effort (middle-size league). His research interests include behavior engineering, robot navigation, omni-directional vision, data interpretation and control for autonomous robotic agents, mobile robot design, reinforcement learning and fuzzy systems.

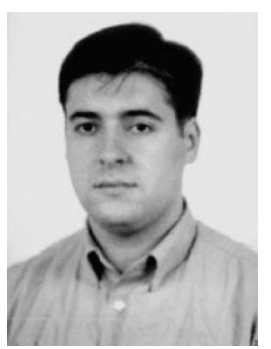

Carlos Machado was born in 1974 in Braga, Portugal. He received his degree in Industrial Electronic Engineering at the University of Minho, Guimarães, Portugal in 1998. Since 1999, he works at the same University. He is currently an M.Sc. student in Industrial Electronics, specialty Robotics. His research interest includes mobile and autonomous robotics, electronics, vision, image processing and simulation.

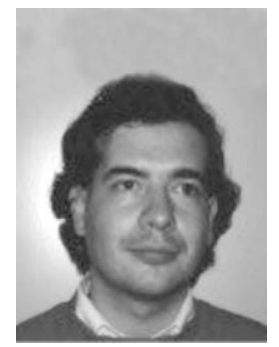

Fabio Marchese is a Research Associate at Dipartimento di Informatica, Sistemistica e Comunicazione, Università degli Studi di Milano - Bicocca. He got his Laurea in Electronic Engineering in October 1991 from the Politecnico di Milano, the Ph.D. in Computer and Control Engineering from Politecnico di Milano in September 1997. His research interest includes robotics and autonomous agents, 3D vision for mobile robot navigation, omni-directional vision, classic and adaptive edge detection, path-planning, cellular automata, learning.

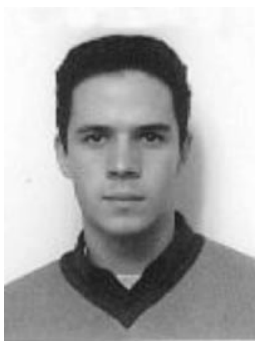

Carlos Marques got his Licenciatura (5 years) and M.Sc. degrees in 1998 and 2001, respectively, both in Electrical and Computer Engineering, from the Instituto Superior Técnico (IST), Lisbon Technical University, Portugal. Since 2001, he is the co-founder of a small Portuguese company (IdMind) which develops educational robots. He is the co-winner of the RoboCup 2000 Workshop Scientific Challenge Award. His current research interests include guidance algorithms and cooperative robotics. 


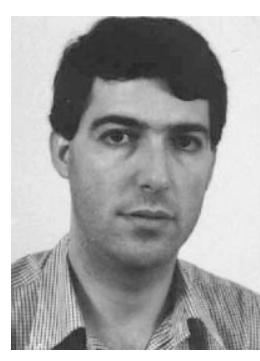

Fernando Ribeiro was born in 1966 in Guimarães, Portugal, studied Computer Science at Portucalense University and received his degree in 1988. In 1991, he moved to England where he studied Industrial Robotics at Cranfield University for the degree of M.Sc., achieved in 1992. From 1992 to 1995 , he continued his studies, receiving his Ph.D. diploma on rapid prototyping using industrial robots. Since 1995, he works at the University of Minho, Guimarães, Portugal, where he is an Auxiliary Professor. His research interests include mobile and autonomous robotics, industrial robotics, vision, image processing and simulation. He is a member of IEEE and IEE.

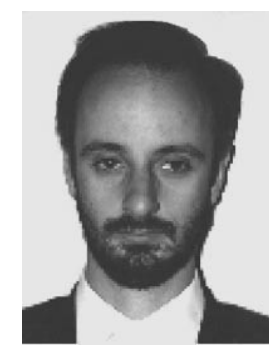

Domenico Sorrenti is an Assistant Professor (ricercatore) with Dipartimento di Informatica, Sistemistica e Comunicazione, Università degli Studi di Milano - Bicocca. He got his Laurea in Electronic Engineering in February 1989 from the Politecnico di Milano, and his Ph.D. in Computer and Control Engineering, from the Politecnico di Milano in December 1992.

His research interests include 3D vision for mobile robot navigation, basic image processing algorithms, adaptive edge detection, omni-directional vision, image-based pedestrian and vehicle traffic monitoring. He is a member of the IEEE Robotics and Automation Society, the IEEE Computer Society, IAPR (International Association for Pattern Recognition), AI*IA (Italian Association for Artificial Intelligence). 